\title{
Reaction of cyclopentadienyl and methyl radicals
}

\author{
Vladislav S. Krasnoukhov ${ }^{1}$, Aleksander M. Mebel ${ }^{2}$, Igor P. Zavershinskiy ${ }^{1}$, \\ and Valeriy N. Azyazov ${ }^{1,3}$ \\ ${ }^{1}$ Samara National Research University, 34 Moskovskoye Shosse, Samara 443086, Russia \\ ${ }^{2}$ Florida International University, SW 8 th St, Miami, FL 33199, USA \\ ${ }^{3}$ Lebedev Physical Institute, Samara, Russia, 221 Novo-Sadovaya Str., Samara 443011, Russia \\ * e-mail: vladya11@gmail.com
}

\begin{abstract}
Geometries and potential energies of reagents, products, and intermediate states for the reaction between cyclopentadienyl $\left(\mathrm{C}_{5} \mathrm{H}_{5}\right)$ and methyl $\left(\mathrm{CH}_{3}\right)$ radicals are found by means of ab initio quantum mechanical methods CCSD(T)/cc-pVTZ-f12, B2PLYPD3/AUG-CC-PVDZ and B3LYP/6-311G. Basing on the analysis of the found energy, structural and kinetic characteristics of the compounds involved, the reaction paths leading to the formation of fulvene and benzene, the simplest aromatic compound, are determined. The reaction path begins from the formation of the intermediate compound, methylcyclopentadiene, followed by tearing-off a hydrogen atom from it: $\mathrm{C}_{5} \mathrm{H}_{5}+\mathrm{CH}_{3} \rightarrow \mathrm{C}_{5} \mathrm{H}_{5} \mathrm{CH}_{3} \rightarrow \mathrm{C}_{5} \mathrm{H}_{4} \mathrm{CH}_{3}+\mathrm{H}$. The subsequent monomolecular transformations of $\mathrm{C}_{5} \mathrm{H}_{4} \mathrm{CH}_{3}$ are closed by the formation of either fulvene (via the loss of one hydrogen atom from the methyl group) or benzene (via the stages of transforming the pentamerous ring into a hexamerous one and tearing-off a hydrogen atom). The rate constants found in the paper using the software package MESS show that the rate of benzene formation is always higher than that for fulvene within the temperature interval 500-2250 K. Since fulvene can also isomerize into benzene, the reaction $\mathrm{C}_{5} \mathrm{H}_{5}+\mathrm{CH}_{3}$ is an important supplier of the initial bricks for building polycyclic aromatic hydrocarbons dangerous for living systems. (C) 2017 Journal of Biomedical Photonics \& Engineering.
\end{abstract}

Keywords: Combustion, methyl, cyclopentadienyl, benzene, fulvene, PANs, quantum chemistry calculations, reaction pathways, rate constant.

Paper \#3131 received 04 Dec 2016; revised manuscript received 12 Mar 2017; accepted for publication 14 Mar 2017; published online 26 Mar 2017. doi: 10.18287/JBPE17.03.020304. [Special Issue. Workshop "Biophotonics" of the XIII all-Russian Youth Samara conference-contest on optics and laser physics].

\section{References}

1. J. D. Bittner, and J. B. Howard, "Composition profiles and reaction mechanisms in a near-sooting premixed benzene/oxygen/argon flame," Symposium (International) on Combustion 18(1), 1105-1116 (1981).

2. P. R. Westmoreland, A. M. Dean, J. B. Howard, and J. P. Longwell, "Experimental and theoretical analysis of oxidation and growth chemistry in a fuel-rich acetylene flame,” J. Phys. Chem. 93, 8171-8180 (1989).

3. I. Glassman (ed.), Combustion, 2nd Edition, Academic Press, New York (1987). ISBN: 0-12-285851-4.

4. A. J. Colussi, F. Zabel, and S. W. Benson, "The very low-pressure pyrolysis of phenyl ethyl ether, phenyl allyl ether, and benzyl methyl ether and the enthalpy of formation of the phenoxy radical," Int. J. Chem. Kinet. 9(2), 161-178 (1977).

5. C.-Y. Lin, and M. C. Lin, "Thermal decomposition of methyl phenyl ether in shock waves: The kinetics of phenoxy radical reactions," J. Phys. Chem. 90(3), 425-431 (1986).

6. C. A. Taatjes, D. L. Osborn, T. M. Selby, G. Meloni, A. J. Trevitt, E. Epifanovsky, A. I. Krylov, B. Sirjean, E. Dames, and H. Wang, "Products of the benzene + O (3P) reaction," J. Phys. Chem. A 114(9), 3355-3370 (2010). 
7. A. Burcat, and M. Dvinyaninov, "Detailed kinetics of cyclopentadiene decomposition studied in a shock tube," Int. J. Chem. Kinet. 29(7), 505-514 (1997).

8. "Hazard Prevention and Control in the Work Environment: Airborne Dust," World Health Organization (2015).

9. M. Frenklach, "Reaction Mechanism of Soot Formation in Flames," PCCP 4(11), 2028-2037 (2002).

10. L. V. Moskaleva, A. M. Mebel, and M. C. Lin, "The CH3 + C5H5 reaction: A potential source of benene at high temperatures," P. Combust. Inst. 26(1), 521-526 (1996).

11. S. Sharma, and W. H. Green, "Computed Rate Coefficients and Product Yields for c-C5H5 + CH3 $\rightarrow$ Products,” J. Phys. Chem. A 113(31), 8871-8882 (2009).

12. C. F. Melius, BAC-MP4 Heats of Formation and Free Energies, Sandia National Laboratories, Livermore, CA (1993).

13. A. W. Jasper, and N. Hansen, "Hydrogen-assisted isomerizations of fulvene to benzene and of larger cyclic aromatic hydrocarbons," Proc. of the Combustion Institute 34(1), 279-287 (2013).

14. L. A. Curtiss, K. Raghavachari, G. W. Trucks, and J. A. Pople, "Gaussian - 2 theory for molecular energies of first - and second - row compounds," J. Chem. Phys. 94(11), 7221-7230 (1991).

15. J. A. Pople, M. Head-Gordon, D. J. Fox, K. Raghavachari, and L. A. Curtiss, "Gaussian - 1 theory: A general procedure for prediction of molecular energies,” J. Chem. Phys. 90(10), 5622-5629 (1989).

16. L. A. Curtiss, C. Jones, G. W. Trucks, K. Raghavachari, and J. A. Pople, "Gaussian - 1 theory of molecular energies for second - row compounds," J. Chem. Phys. 93(4), 2537-2545 (1990).

17. H.-J. Werner, P. J. Knowles, G. Knizia, F. R. Manby, and M. Schütz, "Molpro: a general - purpose quantum chemistry program package," Wiley Interdisciplinary Reviews: Computational Molecular Science 2(2), 242$253(2012)$.

18. Y. Georgievskii, J. A. Miller, M. P. Burke, and S. J. Klippenstein, "Reformulation and Solution of the Master Equation for Multiple-Well Chemical Reactions,” J. Phys. Chem. A 117(46), 12146-12154 (2013).

\section{Introduction}

Polycyclic aromatic hydrocarbons $(\mathrm{PAH})$ comprise a considerable part of harmful emission from different fossil fuel power plants and serve as predecessors in soot formation [1-3]. They hugely affect the environment and human health. Appeared in the air and soil, they can be inhaled by humans or consumed with food, which leads to various health problems. PAH are very toxic by their nature, their prolonged effect is related to such diseases as skin allergy, immune deficit, asthma and other lung diseases, destruction of red blood cells, tumours, preterm birth, diseases of kidneys and liver, and valvular defect in neonates.

Methyl and cyclopentadienyl are among the key radicals in the PAH and soot formation. The methyl radical can be formed by partitioning of complex hydrocarbons or by their interaction with oxygen, while the cyclopentadienyl is turned out in the reactions of benzene and phenyl with atomic oxygen [6], in the pyrolysis of phenoxy radical [3-6], phenol [6], cyclopentadiene [7], etc. The reaction $\mathrm{CH}_{3}+\mathrm{C}_{5} \mathrm{H}_{5}$ is interesting because it yields the first aromatic compound, benzene, that is an initial brick in building more complex compounds, such as PAH, soot, carbon nanoparticles, fullerene, etc. There are strict standards on emission of these compounds for different power plants using hydrocarbon fuels [8]. At present, the search for hydrocarbon fuel burning regimes, in which the formation of aromatic compounds is suppressed basing on the detailed analysis or the processes that occur in the burning zone is carried out [9]. The reaction between methyl radical and cyclopentadienyl occupies an important position among the kinetic reaction schemes of the formation of aromatic compounds.

The reaction of $\mathrm{CH}_{3}$ with $\mathrm{C}_{5} \mathrm{H}_{5}$ was studied in theoretical papers [10-13]. Refs. [10,11] show that the final products of this reaction are fulvene and benzene. Melius et al. [12] theoretically studied the isomerisation of fulvene using the method BAC-MP4. Jasper et al. [13] reported a detailed consideration of the fulvene-tobenzene isomerisation process catalysed by atomic hydrogen. However, in these papers, not all ways leading to the formation of benzene were considered, and no rate constants of elementary processes, depending on temperature and pressure, were presented.

In this paper, we present the geometries of the initial and final products involved in the reaction as well as of the intermediate and transition compounds, calculated by means of the density functional theory (DFT), B2PLYPD3 and B3LYP methods. For all found structures, the energy values are corrected using ab initio quantum chemistry calculations. Basing on the obtained data, we determine the optimal reaction paths for the system $\mathrm{C}_{5} \mathrm{H}_{5}+\mathrm{CH}_{3}$, leading to the formation of benzene and fulvene. Using the corrected energies and optimised geometries, we calculated the rate constants for a number of basin monomolecular reactions in the multistep process of interaction between the two radicals at the temperatures $500-2500 \mathrm{~K}$. 


\section{Methodology}

We used ab initio quantum mechanical methods of high level to study the possible products of the reaction $\mathrm{CH}_{3}+\mathrm{C}_{5} \mathrm{H}_{5}$. The optimised structures and energies of the reagents, products, intermediate and transient states involved in the reaction were found at the first stage using the widespread hybrid method of density functional theory B3LYP with the basis set $6-311 G^{* *}$. Using the same method, we calculated the vibrational frequencies and zero-point energies (ZPE) for all the structures.

At the second stage the energies, the optimised geometries and vibrational frequencies for all structures were found using more up-to-date density functional method B2PLYPD3 with the basis set AUG-CC-PVDZ in order to compare the two methods applied to the system $\mathrm{CH}_{3}+\mathrm{C}_{5} \mathrm{H}_{5}$.

At the last stage the total energies of the involved structures were corrected using the method of couple clusters with the single and double excitations taken into account $(\operatorname{CCSD}(\mathrm{T}))$ with the basis set cc-pVTZ-f12. The used method allows the energy calculations with the accuracy to $1-3 \mathrm{kcal} / \mathrm{mole}$. The B3LYP and B2PLYPD3 calculations were carried out using the Gaussian software packages [14-16], while the $\operatorname{CCSD}(\mathrm{T})$ calculations were performed using the Molpro software package [17].

Basing on the corrected total energies and geometry of reagents, products, intermediate and transient states, we calculated the rate constants at different pressures and temperatures, using the software package MESS, based on the Rice-Ramsperger-Kassel-Marcus statistical theory [18].

\section{Results and discussion}

Figure 1 presents the reaction paths of interaction between the $\mathrm{CH}_{3}$ and $\mathrm{C}_{5} \mathrm{H}_{5}$ radicals. The energies of the transient states $\mathrm{TSi}$, the intermediate states and the products, calculated using three methods, are presented relative to the total energy of the initial reagents in the $\mathrm{kcal} / \mathrm{mole}$ units. As a result of the interaction at the first stage the methylcyclopentadiene $\mathrm{C}_{5} \mathrm{H}_{5} \mathrm{CH}_{3}$ molecule is formed. Further transformations are related to the possible transition to the initial products or with the hydrogen atom tearing-off and the formation of $\mathrm{C}_{5} \mathrm{H}_{4} \mathrm{CH}_{3}$ or $\mathrm{C}_{5} \mathrm{H}_{5} \mathrm{CH}_{2}$ molecules. In Ref. [10] it was shown that the first path is less energy-consuming, therefore, below we will consider the path with the formation of the molecule $\mathrm{C}_{5} \mathrm{H}_{4} \mathrm{CH}_{3}$ (1). Then the path via the transient state (barrier) TS6 with the decay into fulvene $\mathrm{C}_{5} \mathrm{H}_{4} \mathrm{CH}_{2}$ (2) and the hydrogen atom. An alternative path from the structure $\mathrm{C}_{5} \mathrm{H}_{4} \mathrm{CH}_{3}$ is related to regrouping the atoms into the molecule $\mathrm{C}_{5} \mathrm{H}_{5} \mathrm{CH}_{2}$ (3) with the activation barrier TS5 of $54.1 \mathrm{kcal} / \mathrm{mole}$. In further transformations either the structure $\mathrm{C}_{5} \mathrm{H}_{5} \mathrm{CH}_{2}$ yields a fulvene molecule and a hydrogen atom with the transient barrier TS1 of $61.1 \mathrm{kcal} / \mathrm{mole}$, or the expansion of the ring begins leading to the formation of the $\mathrm{C}_{5} \mathrm{H}_{5} \mathrm{CH}_{2} \mathrm{RE}$ (4) structure with the transient barrier TS2 of $37.9 \mathrm{kcal} / \mathrm{mole}$. After passing the barrier TS3 of $40.99 \mathrm{kcal} / \mathrm{mole}$ the bond between carbon atoms is broken and the cyclohexadienyl structure $\mathrm{C}_{6} \mathrm{H}_{6} \mathrm{H}(5)$ is formed. Finally, the hydrogen atom tearing-off leads to the formation of the benzene ring $\mathrm{C}_{6} \mathrm{H}_{6}(6)$ after passing the barrier TS4 $(31.1 \mathrm{kcal} / \mathrm{mole})$. The relative energy of the final state of this chain in the form of fulvene amounts to $56.1 \mathrm{kcal} / \mathrm{mole}$, while in Ref. [10] it was equal to $54.2 \mathrm{kcal} / \mathrm{mole}$. The ab initio method chosen here, providing higher precision as compared with G2M (rcc, MP2) used in Ref. [10], allowed higher accuracy of relative energies of the structures, involved in the reaction (Table 1).

Table 1 Relative corrected energies of reagents, transient states, and reaction products obtained using the method CCSD(T)/cc-pVTZ-f12.

Molecule

Relative energy, kcal/mole

\begin{tabular}{cc}
\hline $\mathrm{CH}_{3}+\mathrm{C}_{5} \mathrm{H}_{5}$ & 0.0 \\
\hline $\mathrm{C}_{5} \mathrm{H}_{5} \mathrm{CH}_{3}$ & -70.4 \\
\hline $\mathrm{C}_{5} \mathrm{H}_{4} \mathrm{CH}_{3}(1)$ & 1.4 \\
\hline $\mathrm{TS} 5$ & 54.1 \\
\hline $\mathrm{C}_{5} \mathrm{H}_{5} \mathrm{CH}_{2}(3)$ & 29.6 \\
\hline $\mathrm{TS} 1$ & 61.1 \\
\hline $\mathrm{TS} 2$ & 37.9 \\
\hline $\mathrm{C}_{5} \mathrm{H}_{5} \mathrm{CH}_{2} \mathrm{RE}(4)$ & 31.5 \\
\hline $\mathrm{C}_{5} \mathrm{H}_{4} \mathrm{CH}_{2}(2)$ & 56.1 \\
\hline $\mathrm{TS}_{3}$ & 41.0 \\
\hline $\mathrm{C}_{6} \mathrm{H}_{6} \mathrm{H}_{(5)}$ & 4.3 \\
\hline $\mathrm{TS}_{4}$ & 31.1 \\
\hline $\mathrm{C}_{6} \mathrm{H}_{6}(6)$ & 24.7
\end{tabular}

Table 2 Rate constants for the reactions $\mathrm{C}_{5} \mathrm{H}_{4} \mathrm{CH}_{3} \rightarrow$ $\mathrm{C}_{5} \mathrm{H}_{4} \mathrm{CH}_{2}, \mathrm{C}_{5} \mathrm{H}_{4} \mathrm{CH}_{3} \rightarrow \mathrm{C}_{6} \mathrm{H}_{6}$ under the pressure of 1 atm.

\begin{tabular}{ccc} 
& \multicolumn{2}{c}{$\mathrm{k}\left(\mathrm{s}^{-1}\right)$} \\
\cline { 2 - 3 } $\mathrm{T}(\mathrm{K})$ & $\mathrm{C}_{5} \mathrm{H}_{4} \mathrm{CH}_{3} \rightarrow \mathrm{C}_{5} \mathrm{H}_{4} \mathrm{CH}_{2}$ & $\mathrm{C}_{5} \mathrm{H}_{4} \mathrm{CH}_{3} \rightarrow \mathrm{C}_{6} \mathrm{H}_{6}$ \\
\hline 500 & $6.01 \times 10^{-13}$ & $3.10 \times 10^{-11}$ \\
\hline 600 & $9.51 \times 10^{-9}$ & $2.64 \times 10^{-7}$ \\
\hline 700 & $9.73 \times 10^{-6}$ & $1.64 \times 10^{-4}$ \\
\hline 800 & $1.79 \times 10^{-3}$ & $1.97 \times 10^{-2}$ \\
\hline 900 & 0.11 & 0.87 \\
\hline 1000 & 2.76 & 16.76 \\
\hline 1125 & 72.74 & 318.91 \\
\hline 1250 & 981.79 & $3.29 \times 10^{3}$ \\
\hline 1375 & $7.94 \times 10^{3}$ & $2.11 \times 10^{4}$ \\
\hline 1500 & $4.26 \times 10^{4}$ & $9.36 \times 10^{4}$ \\
\hline 1650 & $2.07 \times 10^{5}$ & $3.76 \times 10^{5}$ \\
\hline 1800 & $6.93 \times 10^{5}$ & $1.08 \times 10^{6}$ \\
\hline 2000 & $2.29 \times 10^{6}$ & $3.02 \times 10^{6}$ \\
\hline 2250 & $6.62 \times 10^{6}$ & $7.39 \times 10^{6}$
\end{tabular}




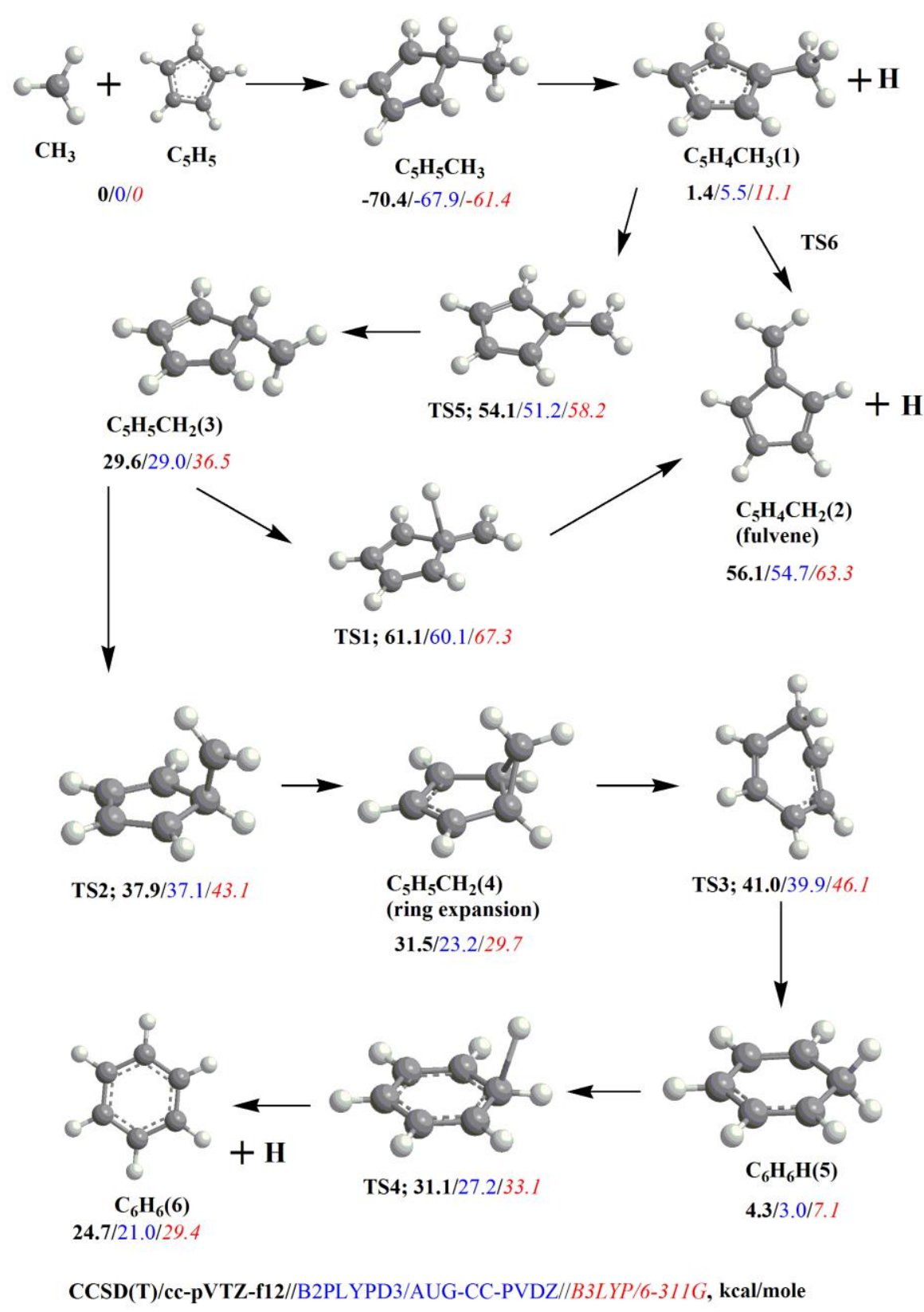

Fig. 1 Reaction paths, structures and relative energies for the reaction of interaction between methyl and cyclopentadienyl radicals. The figures below each structure indicate the relative energy, calculated using the methods $\operatorname{CCSD}(\mathrm{T})$ with the basis set cc-pVTZ-f12 (boldface type), B2PLYPD3 with the basis set AUG-CC-PVDZ (normal type, blue colour), B3LYP with the basis set 6-311G** (italic type, red colour), respectively.

Table 2 presents the rate constants for the sequence of monomolecular transformations from $\mathrm{C}_{5} \mathrm{H}_{4} \mathrm{CH}_{3}$ to $\mathrm{C}_{5} \mathrm{H}_{4} \mathrm{CH}_{2}$ and from $\mathrm{C}_{5} \mathrm{H}_{4} \mathrm{CH}_{3}$ to $\mathrm{C}_{6} \mathrm{H}_{6}$ under the pressure of $1 \mathrm{~atm}$., calculated using the software package MESS [16] that provides kinetic accuracy. From the obtained values, one can see that under the increase of temperature the rates of formation of fulvene and benzene molecules grow exponentially. Under the pressure of $1 \mathrm{~atm}$. the rate of benzene formation exceeds that of the fulvene at all temperatures, considered in the present work. At the temperatures below $1000 \mathrm{~K}$ the rate of monomolecular transformations in the chain $\mathrm{C}_{5} \mathrm{H}_{4} \mathrm{CH}_{3} \rightarrow \mathrm{C}_{6} \mathrm{H}_{6}$ is higher by more than an order of magnitude, while in the temperature interval 1000-2250
$\mathrm{K}$ the rate constants for both reaction paths have the same order of magnitude.

\section{Conclusion}

As a result of the present work, the $\mathrm{CH}_{3}+\mathrm{C}_{5} \mathrm{H}_{5}$ reaction paths are found, the energies of the compounds are determined with higher accuracy, the general picture of fulvene and benzene formation is considered, and the rate constants for the reactions $\mathrm{C}_{5} \mathrm{H}_{4} \mathrm{CH}_{3} \rightarrow \mathrm{C}_{5} \mathrm{H}_{4} \mathrm{CH}_{2}$, $\mathrm{C}_{5} \mathrm{H}_{4} \mathrm{CH}_{3} \rightarrow \mathrm{C}_{6} \mathrm{H}_{6}$ at different temperatures and the pressure of $1 \mathrm{~atm}$. are calculated for the first time. Basing on the analysis of the obtained energy and kinetic characteristics, three reaction paths were 
revealed, two of them leading to the formation of fulvene and one to the formation of benzene. The rate of benzene formation is always higher than that of fulvene over the entire temperature range implemented in combustion chambers. Fulvene is known to be capable of further isomerisation into benzene [13], which makes the reaction $\mathrm{CH}_{3}+\mathrm{C}_{5} \mathrm{H}_{5}$ a potential source of $\mathrm{PAH}$ in combustion chambers of different power plants using hydrocarbon fuel.

The balance between the accuracy and the available computation facilities is commonly achieved by using two-level calculations, where the geometry of reagents, transient and intermediate states are found using the DFT and their energies in optimised geometries are calculated using the $\operatorname{CCSD}(\mathrm{T})$ method. The DFT method B3LYP is most frequently used to find the structural parameters of the compounds at the first level. However, the DFT methods developed in recent years allow the calculation of geometry and vibrational frequencies with the similar or somewhat better accuracy than B3LYP, in particular, the method B2PLYPD3 that allows for the dispersion corrections and, therefore, describes distant interactions better than B3LYP. As follows from our data, presented in Fig. 1, the values of energies calculated using B2PLYPD3 are in much better agreement with those calculated using the $\mathrm{ab}$ initio method than the results of B3LYP. Thus, our data show that the DFT method B2PLYPD3 has successfully passed the test in application to the particular system $\mathrm{CH}_{3}+\mathrm{C}_{5} \mathrm{H}_{5}$.

The present work was supported by the Government of Russian Federation, grant No. 14.Y26.31.0020. 